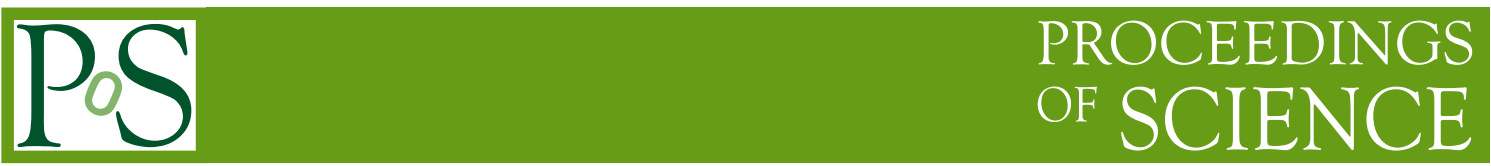

\title{
Phase1 upgrade of the CMS-HF Calorimeter
}

\author{
Erhan Gülmez ${ }^{* \dagger}$ \\ On behalf of the CMS-HCAL Collaboration \\ Bogazici University, Bebek, Istanbul, 34342, Turkey \\ E-mail: gulmez@boun. edu.tr
}

\begin{abstract}
In this presentation, results of the Phase I upgrade of the CMS Hadron Forward Calorimeter (HF) are discussed. The CMS-HF Calorimeter was using regular PMTs. Cherenkov light produced in the quartz fibers embedded in the iron absorber was read out with the PMTs. However, occasionally, stray muons hitting the PMT windows cause Cherenkov radiation in the PMT itself and produce large signals. These large signals mimic a very high-energy particle and are tagged as important by the trigger. To reduce this problem, PMTs had to be replaced. The four-anode PMTs that were chosen have thinner windows; thereby reducing the Cherenkov radiation in the PMT window. As part of the upgrade, the read-out electronics is to be replaced so that the PMTs are read out in two channels by connecting each pair of anodes to a single channel. Information provided by these two channels will help us reject the false signals due to the stray muons since the Cherenkov radiation in the PMT window is more likely to produce a signal only in one anode as opposed to a real signal whose light illuminates all four anodes.
\end{abstract}

Fourth Annual Large Hadron Collider Physics

13-18 June 2016

Lund, Sweden

\footnotetext{
* Speaker.

${ }^{\dagger}$ This work was supported by Turkish Atomic Energy Authority (TAEK) and Bogazici University Research Fund Grant Number: 9620 (15B03P1).
} 


\section{Introduction}

The CMS-HF calorimeter is designed to detect forward jets and particles scattered in the region, $3<\eta<5$. The two units placed at each end of the CMS detector are in cylindrical shape with an active radius of $1.4 \mathrm{~m}$ and $1.65 \mathrm{~m}$ long. Active elements in the calorimeters are plastic-clad quartz fibers. Long $(1.65 \mathrm{~m})$ fibers are sensitive for both EM and hadronic showers while short $(1.43 \mathrm{~m})$ fibers are hadronic showers only. Particles passing through the fibers produce Cherenkov radiation inside the fiber. The light produced by the Cherenkov radiation is carried by the fibers to the PMTs and transferred to them through an arrangement of an air-core light-guide.

\section{Problems During Run I: Anomalous or Window events}

Stray muons passing through the PMTs attached to the HF calorimeter produce Cherenkov radiation right at the window glass of the PMTs, causing false triggers to mimic very high-energy events. Compared to the regular signals produced by the Cherenkov light coming through the fibers, these signals are larger and faster by a few nanoseconds. Initially, the correlation between the electromagnetic and hadronic channel is utilized to remove these unwanted events. Real events are expected to produce signals simultaneously in both electromagnetic and hadronic channels, but not the anomalous events since they are produced right at the window of a specific PMT. However, increasing luminosity will increase the occupancy rate of the channels and the number of muons passing through the PMTs. Simple comparison of the two channels will not be enough to identify the window events.
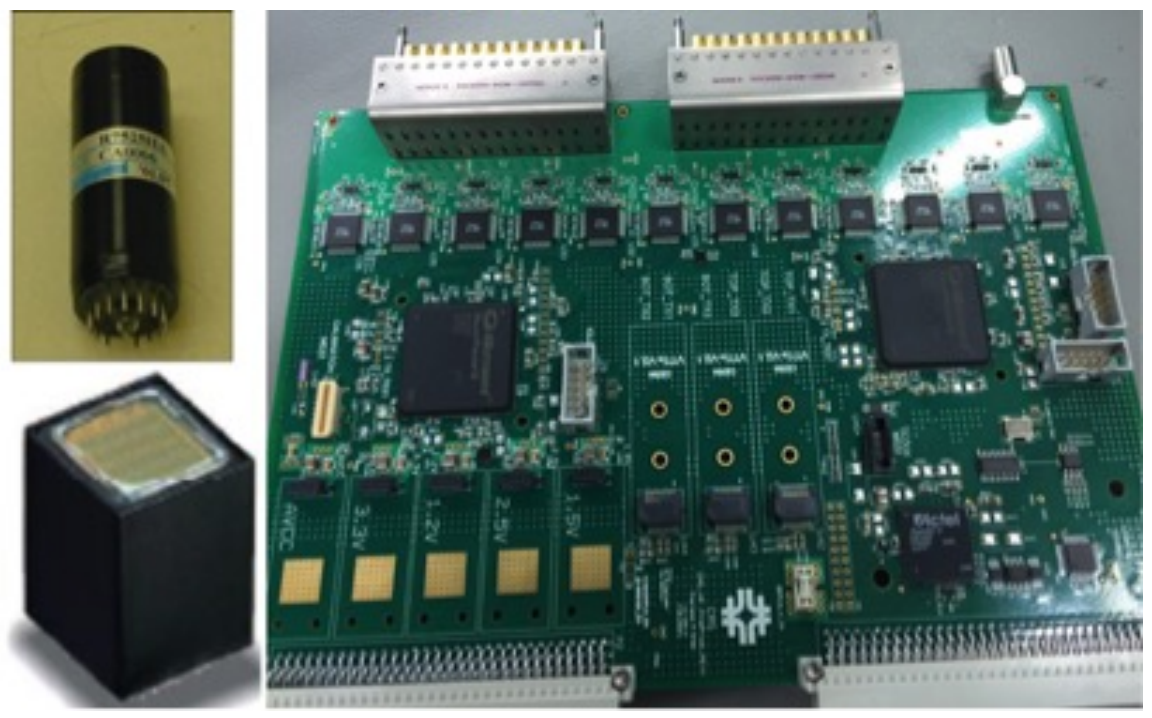

Figure 1: (left-top) Old PMTs: R7525; Bialkali photocathode; Max. 25\% quantum efficiency (QE); Typical gain: $5 \times 10^{5}$; Window: $1.2 \mathrm{~mm}$ thick at the center, $6 \mathrm{~mm}$ thick at the edges; plano-convex geometry with face area $\sim 490 \mathrm{~mm}^{2}$; Glass jacket. (left-bottom) 4-anode PMTs: R7600U-200-M4; Ultra Bialkali photocathode; Max. 43\% QE (350 nm); Typical gain: 1.3×106; Window: <1 mm thick; square geometry with area $\sim 324 \mathrm{~mm}^{2}$; Metal jacket. (right) A picture of the new HF Front-End Electronic Cards with the new QIE10 chips. 


\section{Solution}

Replacing the old PMTs with thinner window 4-anode PMTs (Fig. 1-lower left) decreased the pulse height of the Cherenkov signals produced on the window glass and provided additional channels to eliminate the window events. Regular Cherenkov light coming through the fibers illuminates all four anodes but the Cherenkov light generated at the window is less likely to do so $[1,2,3]$.

During LS1 period, the old PMTs in the HF calorimeters were replaced with the four-anode PMTs as part of the Phase I upgrade project. At the same time the electronic readout cards have been redesigned to read out the new PMTs in two channels and also to provide the timing signal. Until these cards are produced and installed, the new PMTs are read-out as single channel by combining the signals from all four anodes. New 4-anode PMTs almost double the signal for the real events but produce significantly lower level signals for the window events (Fig. 2).
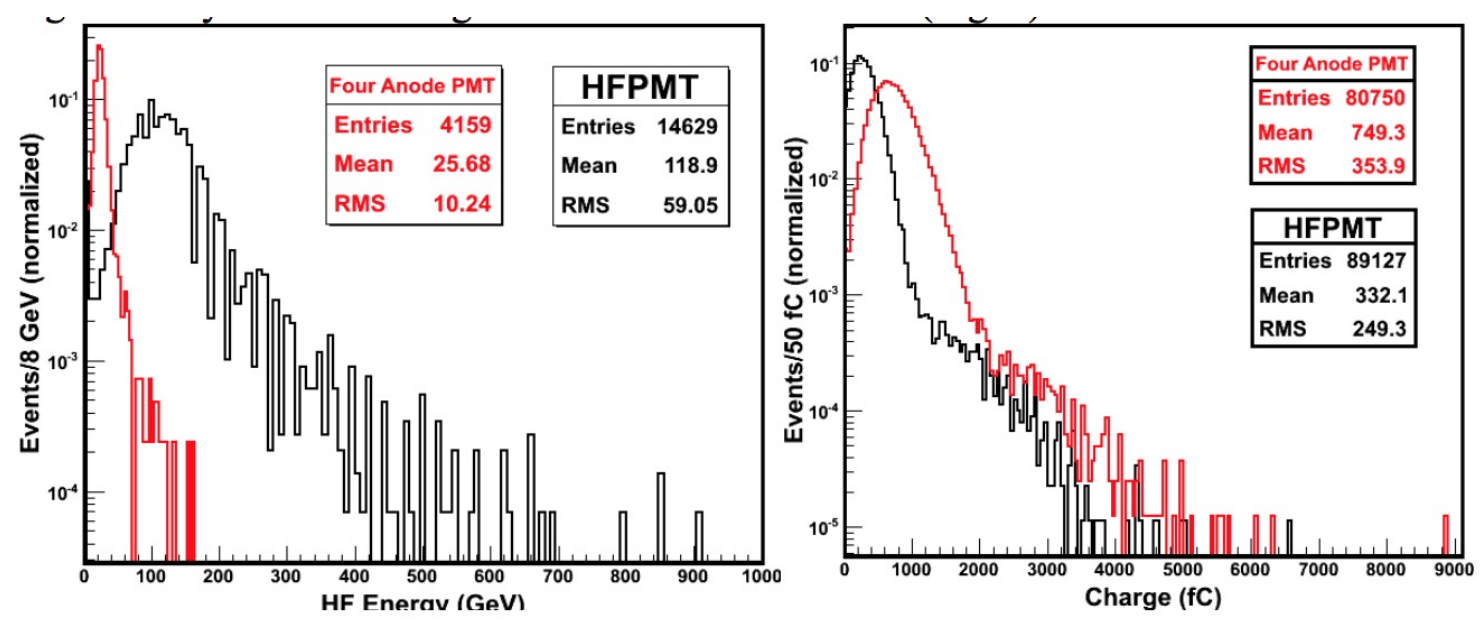

Figure 2: Comparison of signals from the old PMTs (black) and new PMTs (red). Window events are reduced in the new PMTs (left) but the response to the real events is increased (right) $[1,2]$.

\section{HF Front End Electronics cards}

The HF Calorimeter Front-End Electronic cards are redesigned to read out the four-anode PMTs in two channels. These two-channel readout cards also include the new version of the ADC chip (QIE10 instead of QIE8) to provide the timing information for the arrival of the signals. 200 of these cards (Fig. 1-right) were assembled by a Turkish company in Istanbul (SIMPRO) last summer and will be installed at the end of this year during the year-end technical stop (YETS).

\section{Timing in 4-anode PMTs and 2-channel readout}

One of the existing HF-FE cards on the detector has been replaced by a new redesigned card towards the end of the 2015 run period. Timing information obtained from the QIE10 chips on the new card shows that the window events are large events (ADC vs TDC plot - Fig. 3-left) and arrive at an earlier time (TDC histogram - Fig. 3-right) in the event (inside the circled area in both plots) [4]. 


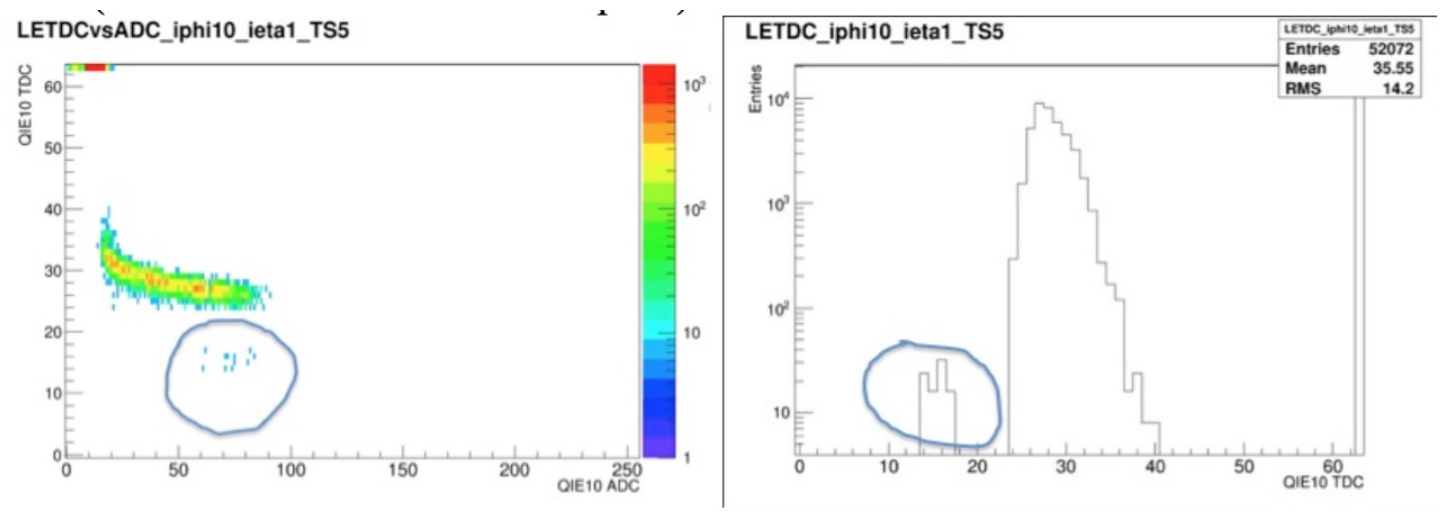

Figure 3: Arrival time of the PMT signals versus their amplitudes obtained (left) and histogram of the arrival time of the PMT signals (right) during the collisions at $13 \mathrm{TeV}$. Circled events in both histograms are window events and they have higher amplitudes and arrive earlier.
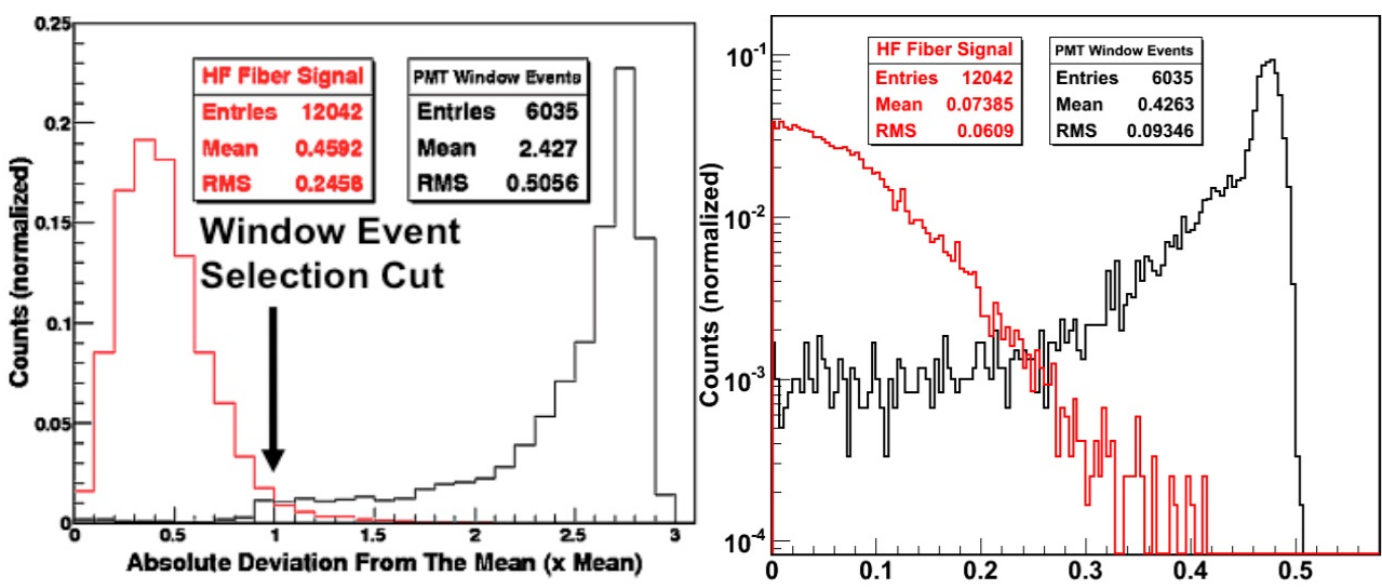

Figure 4: (a) Distributions of the deviation of the single quadrant signal from the mean in multiples of the mean. (b) Distributions of the deviation of maximum signal of the two channels from the mean in multiples of the total signal $[1,2,5]$.

\section{Conclusion: Algorithms to reject the anomalous events}

Even with these improvements on the PMTs, elimination of the window events become difficult as the luminosity further increases. Additional ways to identify the window events have to be devised. Comparison of the individual anode signals may separate the window events from the regular events. To develop some algorithms to eliminate the window events, deviation of the single quadrant signal from the mean of all four quadrants is measured at a test beam study at CERN. HF calorimeter signal is mimicked by using a fiber bundle behind a steel absorber and the fibers are attached to the PMTs. Window events are simulated by placing the PMTs in the muon beam. Distributions of the deviation of the single quadrant signal from the mean in multiples of the mean show a clear separation between two types of events. The plot in Figure $4 \mathrm{a}$ is for four channel 
readout but two channel readout also shows a good separation (Fig. 4b) [1, 2, 5]. It is more feasible and affordable to read out the four-anode PMTs in two channels. Signals from two anodes are combined and read out as a single signal. These algorithms will be studied and developed further once the new cards are installed on the detectors.

\section{References}

[1] Ph.D. Thesis, Burak Bilki, University of Iowa, unpublished, (2011).

[2] CMS HCAL Collaboration, "Study of various photomultiplier tubes with muon beams and Cherenkov light produced in electron showers," J. of Instrumentation, 5, P06002, (2010).

[3] E. Gülmez, "The CMS Hadron Forward Calorimeter Upgrade during Phase I", Proceedings of 14th ICATTP Astroparticle, Particle, Space Physics and Detectors for Physics Applications, 23-27 September, 2013, Como, Italy, 2013, World Scientific, 568(2014).

[4] V. Krishtenko, private communication.

[5] CMS HCAL Collaboration, "Tests of CMS hadron forward calorimeter upgrade readout box prototype", J. of Instrumentation, 7, P10015, (2012). 